ELECTRONIC RESEARCH ANNOUNCEMENTS OF THE AMERICAN MATHEMATICAL SOCIETY

Volume 9, Pages 94-98 (October 8, 2003)

S $1079-6762(03) 00116-1$

\title{
A STRONGLY DIAGONAL POWER OF ALGEBRAIC ORDER BOUNDED DISJOINTNESS PRESERVING OPERATORS
}

\author{
KARIM BOULABIAR, GERARD BUSKES, AND GLEB SIROTKIN
}

(Communicated by Svetlana Katok)

\begin{abstract}
An order bounded disjointness preserving operator $T$ on an Archimedean vector lattice is algebraic if and only if the restriction of $T^{n !}$ to the vector sublattice generated by the range of $T^{m}$ is strongly diagonal, where $n$ is the degree of the minimal polynomial of $T$ and $m$ is its 'valuation'.
\end{abstract}

\section{INTRODUCTION}

Consider a square matrix $T$ for which on every row there is at most one nonzero entry. Let $n$ be the degree of its minimal polynomial and let $m$ be its 'valuation', i.e. the multiplicity of 0 as a root of that minimal polynomial. Then $T^{n !}$ is diagonal, when restricted to the range of $T^{m}$. The latter looks surprising and one suspects that the result is known, but we have not been able to locate a reference for it. In this paper we offer a wide ranging generalization of this matrix result. The condition above simply states that the matrix represents an operator that preserves disjointness in the pointwise ordering. The question arises naturally, as to whether general operators on vector lattices that preserve disjointness behave in a similar fashion. For obvious reasons, when leaving the domain of finite-dimensional spaces, some form or another of continuity is reasonably imposed on the operators considered. Thus we study order bounded disjointness preserving operators on Archimedean vector lattices. Such operators exhibit a remarkable diversity. Weighted composition operators, and the classical shift are all order bounded disjointness preserving operators, while their theory motivates research for stochastic and doubly stochastic operators. From the previous examples, a shift operator behaves differently from the matrix case, whichever diagonal is considered. However, there is a concept of diagonal such that, surprisingly, order bounded disjointness preserving operators that satisfy a polynomial equation do behave like the matrix case. Indeed, the algebraic orthomorphisms serve the role of diagonals. This brings us to the main topic of our paper, algebraic order bounded disjointness preserving operators.

Received by the editors June 18, 2003.

2000 Mathematics Subject Classification. Primary 47B65, 06F20, 06F25.

Key words and phrases. Algebraic, disjointness preserving, locally algebraic, minimal polynomial, orthomorphism, strongly diagonal.

The first and the second authors gratefully acknowledge support from the NATO Collaborative Linkage Grant \#PST.CLG.979398. The second author also acknowledges support from the Office of Naval Research Grant \#N00014-01-1-0322. 
Over the last two decades order bounded disjointness preserving operators have been studied in various directions, such as, their multiplicative representations on functions spaces ([2], [4], [8], [10]), their spectral theory ([5], [6], [14]), and their different polar decompositions ([2], [7], [9], [14]). In this paper we are interested in algebraic order bounded disjointness preserving operators and their connection with what we will call strongly diagonal operators, i.e. step functions of the identity operator [3]. Our main result announces that for every algebraic order bounded disjointness preserving operator $T$ on an Archimedean vector lattice, the restriction of $T^{n !}$ to the vector sublattice generated by the range of $T^{m}$ is a strongly diagonal operator, where $n$ is the degree of the minimal polynomial of $T$ and $m$ is its 'valuation'. Also, we show that the strongly diagonal operators are precisely the algebraic orthomorphisms. The latter explains how our study ties in with the recent paper [12] by Luxemburg, de Pagter and Schep, who for Dedekind complete vector lattices, define the order projections of order bounded operators onto the band of central orthomorphisms to be their diagonals. Finally, we point out that the study of algebraic and locally algebraic operators was initiated by Kaplansky [11], to which we refer for more about these operators.

As a consequence, we find that the absolute value of an algebraic order bounded disjointness preserving operator is algebraic as well, which contrasts with the absolute value of finite rank or compact operators.

Full proofs of the results of this note will appear elsewhere. We use the recent book [1], as well as [3] and [14 as a starting point and we refer the reader to these monographs for unexplained terminology and notation.

\section{Strongly DiAgOnAL OPERATORS AND ALGEBRAIC ORTHOMORPHISMS}

Throughout this section, $L$ denotes an Archimedean vector lattice. Though we assume $L$ to be a real vector lattice, the results in this paper are valid for complex vector lattices as well. Denote by $\operatorname{Orth}(L)$ the lattice ordered algebra of all orthomorphisms on $L$ and by $\operatorname{Orth}_{a}(L)$ the set of algebraic orthomorphisms on $L$. The latter has the structure of a lattice ordered algebra itself, as is further explained in the next theorem.

Theorem 1. An operator on $L$ is strongly diagonal if and only if it is an algebraic orthomorphism. In particular, $\operatorname{Orth}_{a}(L)$ is a lattice ordered subalgebra of $\operatorname{Orth}(L)$.

The composition of two algebraic orthomorphisms is again an algebraic orthomorphism. The latter is not the case in general for algebraic order bounded disjointness preserving operators, though the composition of two order bounded disjointness preserving operators is again an order bounded disjointness preserving operator. A counterexample is provided by the two algebraic order bounded disjointness preserving operators $R$ and $S$ on the vector lattice $C([0,1])$ of all realvalued continuous functions on $[0,1]$ defined by

$$
R(f)(x)=w(x) f(1-x) \text { and } S(f)(x)=f(1-x)
$$

for every $f \in C([0,1])$ and $x \in[0,1]$, where $w(x)=1+x$ if $x \in[0,1 / 2]$ and $w(x)=9(2-x)^{-1} / 4$ if $x \in[1 / 2,1]$.

From the start of the study of algebraic operators by Kaplansky in [11], locally algebraic operators were a constant companion. In particular, Kaplansky's result that the two notions coincide on Banach spaces has been influential. In the following 
we will denote by $\operatorname{Orth}_{\ell a}(L)$ the set of all locally algebraic orthomorphisms on $L$. We give a characterization of the latter set next.

Corollary 1. An orthomorphism on $L$ is locally algebraic if and only if its restriction to every principal band of $L$ is strongly diagonal. In particular, $\operatorname{Orth}_{\ell a}(L)$ is a lattice ordered subalgebra of $\operatorname{Orth}(L)$.

Whereas algebraic orthomorphisms are strongly diagonal, the same is not true for locally algebraic orthomorphisms, even if $L$ is Dedekind complete. Indeed, consider the Dedekind complete vector lattice $c_{00}$ of all real sequences that are zero outside a finite set and the orthomorphism $T$ defined on $c_{00}$ by $T(u)=\left(n u_{n}\right)_{n \in \mathbb{N}}$ for every $u=\left(u_{n}\right)_{n \in \mathbb{N}} \in c_{00}$. One can prove that $T$ is locally algebraic, but not strongly diagonal. The observation that needs to be made here is that the vector lattice $c_{00}$ is not Kaplansky complete, a concept that is introduced by us next.

Definition 1. We say that $L$ is Kaplansky complete if, for every infinite countable set $\mathcal{F}$ of $L$, there exists $f \in L$ and an infinite subset $\mathcal{G}$ of $\mathcal{F}$ such that inf $(f, g) \neq 0$ for every $g \in \mathcal{G}$.

Noticing that every Banach lattice, every $\sigma$-laterally complete vector lattice, and every vector lattice with weak order unit is Kaplansky complete, we next observe that Definition 1 is crucial for understanding when every locally algebraic orthomorphism is strongly diagonal.

Theorem 2. The equality $\operatorname{Orth}_{\ell a}(L)=\operatorname{Orth}_{a}(L)$ holds (and hence every locally algebraic orthomorphism on $L$ is strongly diagonal) if and only if $L$ is Kaplansky complete.

On the other hand, for $\sigma$-Dedekind complete vector lattices, the condition that every orthomorphism is strongly diagonal is very strong as we can see next.

Corollary 2. Assume that $L$ is $\sigma$-Dedekind complete. Then every orthomorphism on $L$ is strongly diagonal if and only if $L$ is finite-dimensional. In particular, every order bounded disjointness preserving operator on $L$ is strongly diagonal if and only if $L$ is finite-dimensional.

Without the assumption that $L$ is $\sigma$-Dedekind complete, Corollary 2 need not hold. Indeed, for the $\sigma$-Dedekind complete vector lattice $L$ of all real-valued continuous piecewise affine functions on $[0,1]$ we have that $\operatorname{Orth}(L)=\mathbb{R} . I$, where $I$ is the identity operator of $L$. Hence, all orthomorphisms are strongly diagonal, while $L$ is infinite-dimensional. Also, we cannot replace 'strongly diagonal' by 'locally algebraic'. It suffices to see that $\operatorname{Orth}\left(c_{00}\right)$ coincides with the vector lattice of all real sequences and thus all of the orthomorphisms on $c_{00}$ are locally algebraic.

\section{Algebraic order Bounded Disjointness PRESERVING OPERATORS AND STRONGLY DIAGONAL OPERATORS}

In this section, instead of orthomorphisms, we consider the more general setting of order bounded disjointness preserving operators. In fact, this section includes the central result of our paper. Its proof is based on the existence of invariant principal order ideals, the Kakutani representation theorem and the representation of order bounded disjointness preserving operators on spaces of the type $C(\Omega)$ as weighted composition operators [5]. From now on, $T$ denotes an order bounded disjointness 
preserving operator on the Archimedean vector lattice $L$. Let us recall that the 'valuation' of a polynomial is the multiplicity of 0 as a root of that polynomial.

Theorem 3. The order bounded disjointness preserving operator $T$ is algebraic if and only if there exist natural numbers $m$ and $n$, with $n>m$, such that the restriction of $T^{n !}$ to the vector sublattice of $L$ generated by the range of $T^{m}$ is strongly diagonal. Furthermore, if $T$ is algebraic, then $n$ (respectively, $m$ ) can be chosen as the the degree (respectively, the 'valuation') of the minimal polynomial of $T$.

It follows that the absolute value of an algebraic order bounded disjointness preserving operator is algebraic as well. This seems far from obvious without the representation in Theorem 3 and contrasts with the fact that the absolute value of a finite rank operator need not be a finite rank operator [1. We recall here that the absolute value of an order bounded disjointness preserving operator exists and is a positive disjointness preserving operator [13. If we make some additional assumptions, we can improve the conclusion of Theorem 3 considerably. We give two examples of such additional conditions, each of them is of independent interest for, e.g., spectral theory.

Corollary 3. Assume that $T$ is surjective or injective. Then $T$ is algebraic if and only if there exists $n \in \mathbb{N}$ such that $T^{n !}$ is strongly diagonal. Moreover, $n$ can be chosen to be the degree of the minimal polynomial of $T$.

Corollary 4. If $T$ is order continuous and algebraic with order dense range in $L$, then $T^{n !}$ is strongly diagonal, where $n$ is the degree of the minimal polynomial of $T$. If $L$ is in addition Dedekind complete, then, even without the condition of order continuity, there exists $R \in \operatorname{Orth}(L)$ and $S \in Z(L)$ such that $R=S T^{n !}$, where $Z(L)$ denotes the centre of $L$.

We remark, at the end of this paper, that the finite-dimensional version of Theorem 3 was actually the starting point of this paper.

\section{REFERENCES}

[1] Yu. A. Abramovich, C. D. Aliprantis, An Invitation to Operator Theory, Graduate Studies Math., Vol. 50, Amer. Math. Soc., Providence, RI, 2002. MR 2003h:47072

[2] Yu. A. Abramovich, A. K. Kitover, Inverses of disjointness preserving operators, Memoirs Amer. Math. Soc. 143 (2000), no. 679. MR 2000k:47047

[3] C. D. Aliprantis, O. Burkinshaw, Positive Operators, Academic Press, Orlando, FL, 1985. MR 87h:47086

[4] J. Araujo, E. Beckenstein, L. Narici, Biseparating maps and homeomorphic real-compactifications, J. Math. Ana. Appl. 12 (1995), 258-265. MR 96b:46038

[5] W. Arendt, Spectral properties of Lamperti operators, Indiana Univ. Math. J. 32 (1983), 199-215. MR 85d:47040

[6] W. Arendt, D. R. Hart, The spectrum of quasi-invertible disjointness preserving operators, J. Funct. Anal. 33 (1986), 149-167. MR 87j:47051

[7] K. Boulabiar, G. Buskes, Polar decomposition of order bounded disjointness preserving operators, Proc. Amer. Math. Soc., To appear.

[8] K. Boulabiar, G. Buskes, M. Henriksen, A generalization of a theorem on biseparating maps, J. Math. Ana. Appl. 280 (2003), 336-351.

[9] J. J. Grobler, C. B. Huijsmans, Disjointness preserving operators on complex Riesz spaces, Positivity 1 (1997), 155-164. MR 99k:47079

[10] K. Jarosz, Automatic continuity of separating linear isomorphisms, Bull. Canadian Math. Soc. 33 (1990), 139-144. MR 92j:46049 
[11] I. Kaplansky, Infinite Abelian Groups, University of Michigan Press, Ann Arbor, 1954. MR 16:444g

[12] W. A. J. Luxemburg, B. de Pagter, A. R. Schep, Diagonals of the powers of an operator on a Banach lattice, Operator theory in function spaces and Banach lattices, Oper. Theory Adv. Appl., Vol. 75, Birkhäuser, 1995, pp. 223-273. MR 97i:47076

[13] M. Meyer, Les homomorphismes d'espaces vectoriels réticulés complexes, C. R. Acad. Sci. Paris, Serie. I 292 (1981), 793-796. MR 82f:47051

[14] P. Meyer-Nieberg, Banach Lattices, Springer-Verlag, Berlin, 1991. MR 93f:46025

IPEST, Université de Carthage, BP 51, 2070-La Marsa, Tunisia

E-mail address: karim.boulabiar@ipest.rnu.tn

Department of Mathematics, University of Mississippi, MS 38677

E-mail address: mmbuskes@olemiss.edu

Department of Mathematics, Northern Illinois University, DeKalb, IL 60115

E-mail address: sirotkin@math.niu.edu 